\title{
Electronic and Optical Properties of Rocksalt CdO: A first-Principles Density-Functional Theory Study
}

\author{
Gang Yao ${ }^{1,2}$, Xinyou $\mathrm{An}^{1}$, Hongwen Lei ${ }^{1}$, Yajun $\mathrm{Fu}^{1}$, Weidong $\mathrm{Wu}^{1,2}$ \\ Science and Technology on Plasma physics Laboratory, Research Center of Laser Fusion, CAEP, P.O.Box 919-983, Mianyang \\ 621900, P.R. China \\ State Key Laboratory Cultivation Base for Nonmetal Composites and Functional Materials, School of Materials Science and \\ Engineering, Southwest University of Science and Technology, Mianyang 621010, P.R. China \\ Email: wuweidongding@163.com
}

Received 2012

\begin{abstract}
The structural, electronic and optical properties of rocksalt $\mathrm{CdO}$ have been studied using the plane-wave-based pseudopotential density functional theory within generalized gradient approximation. The calculated lattice parameters are in agreement with previous experimental work. The band structure, density of states, and Mulliken charge population are obtained, which indicates that rocksalt $\mathrm{CdO}$ having the properties of a halfmetal due to an indirect band gap of $-0.51 \mathrm{eV}$. The mechanical properties show that rocksalt $\mathrm{CdO}$ is mechanically stable, isotropic and malleable. Significantly, we propose a correct value for $\varepsilon_{1}(0)$ of about 4.75 , which offers theoretical data for the design and application for rocksalt $\mathrm{CdO}$ in optoelectronic materials.
\end{abstract}

Keywords: Density-Functional Theory; Electronic Structure; Optical Properties; Rocksalt CdO

\section{Introduction}

Recently, electronic structure and optical properties of transparent conductive oxides (TCOS) such as cadmium oxide $(\mathrm{CdO})$ are of tremendously increasing interest, in response to the industrial demand for semiconductor photoelectric device operating in solar cell, liquid-crystal displays (LCD), gas sensor, electrochromic devices and ultraviolet semiconductor laser $[1,2]$. CdO has various polymorphs such as $\operatorname{Pm} 3 m\left(O_{h}^{1}\right), F-43 m\left(T_{d}^{2}\right)$ and $P 6_{3} m c\left(C_{6 v}^{4}\right)$, and it crystallizes in the rocksalt structure with $\mathrm{Fm}-3 \mathrm{~m}\left(\mathrm{O}_{h}^{5}\right)$ space group at room temperature. However, on applying pressure up to 89GPa [3] (90.6 GPa [4]), rocksalt $\mathrm{CdO}$ undergoes a structural phase transition to CsCl-type with $\operatorname{Pm} 3 m\left(O_{h}^{1}\right)$.

Several experimental as well as quite a few ab initio theoretical studies involving method of local density approximation (LDA) [5], Hartree-Fock method [6], full potential linearized augmented plane wave (FP-LAPW) [3] and other calculations based on density functional theory (DFT) have been performed to study the elastic and electronic properties of the rocksalt $\mathrm{CdO}$ over the past two decades [2,3,5-9]. Schleife et al. have reported the complex dielectric function and predicted a static dielectric function $\varepsilon_{1}(0)$ of 7.20 [5]. As we all known, however, the DFT-GGA scheme tends to underestimate slightly the bonding in the considered group-II oxide polymorphs $[3,5]$, i.e., the prediction for $\varepsilon_{1}(0)$ is not reliable.

In order to understand the relevant phenomena and design process of new materials, it is necessary to insight into the electronic and optical properties of rocksalt $\mathrm{CdO}$ by the theoretical analysis. So we have made a correction to optical with scissors operator [10] on the basic of the calculated band gap. And many useful results have been obtained, which offers theoretical data for the design and application for rocksalt $\mathrm{CdO}$ in optoelectronic materials.

\section{Computational Details}

The ab initio calculations were performed by employing plane-wave ultrasoft pseudopotential and implemented in the most recent version of CASTEP code [11]. The exchange and correlation function were given by generalized gradient approximation (GGA) with Perdew-Burke-Ernzerhof , and Perdew and Wang (PW91) [12]. The valence-electron configurations for the elements of rocksalt $\mathrm{CdO}$ are $\mathrm{Cd} 4 \mathrm{~d}^{10} 5 \mathrm{~s}^{2}$ and $\mathrm{O} 2 \mathrm{~s}^{2} 2 \mathrm{p}^{4}$. The cutoff energy of $600 \mathrm{eV}$ was employed through-out the calculation which was tested to be fully converged with respect to total energy for different volumes. The Brillouin-zone sampling mesh parameters for the k-point set were $6 \times 6 \times 6$ [13]. In addition, this set of parameters assures were implemented: the total energy tolerance of 
$5 \times 10^{-6} \mathrm{eV} /$ atom, the maximum force of $0.01 \mathrm{eV} / \AA$, the maximum stress of $0.02 \mathrm{GPa}$ and the maximum displacement of $5 \times 10^{-4} \AA$.

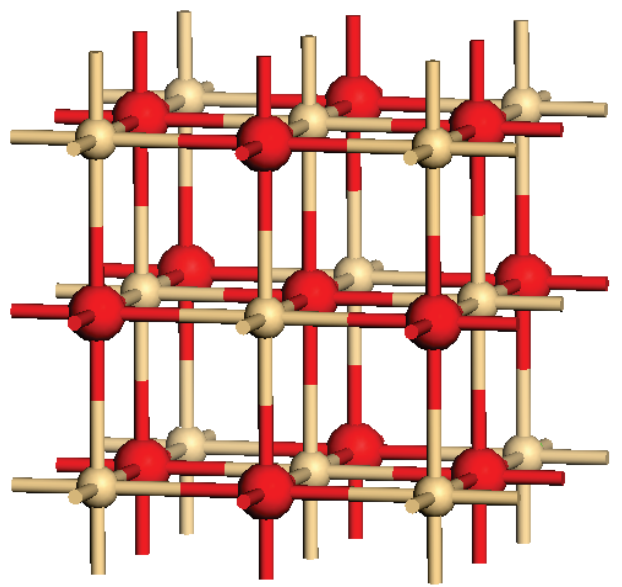

Figure 1. (Color online) Crystal structure of rock-salt $\mathrm{CdO}$ with space group $\mathrm{Fm}-3 \mathrm{~m}\left(\mathrm{O}_{h}^{5}\right.$, SG 225).All atoms are in full occupancy. The pink (small light gray) and the red (dark gray) ball are cadmium and oxygen atom, respectively.

The crystal structure of CdO with Fm-3m $\left(O_{h}^{5}\right)(\# 225)$ space group [14] is shown in Figure 1. It has the following atoms position. $\mathrm{Cd}$ : $4 \mathrm{a}(0,0,0)$; and $\mathrm{O}: 4 \mathrm{~b}(1 / 2,1 / 2$, $1 / 2$ ). The lattice parameters of rocksalt $\mathrm{CdO}$ were optimized in this work by using first-principle calculations and the optimized lattice parameters are compared with the experimental data in Table1.

Table 1. Calculated lattice parameters $a$ (in Å) compared with available experimental data $[4,15]$ for rocksalt $\mathrm{CdO}$.

\begin{tabular}{lllll}
\hline & PBE & PW91 & Expt. [4] & Expt. [15] \\
\hline$a(\AA)$ & 4.797 & 4.792 & 4.770 & 4.777
\end{tabular}

It is obvious that both PBE and PW91 could handle the exchange correlation potential, whereas the lattice parameter calculated by PW91 is more in good agreement with previous experimental conclusion. Hence, all following work based on PW91.

\section{Results and discussion}

\subsection{Electronic and chemical bonding}

The band structure and density of states of rocksalt $\mathrm{CdO}$ are shown in Figures 2 and 3. The valence band maximum is taken as the zero of energy. It is clear that the direct band gap appears at the $\Gamma$ point with energy of $0.55 \mathrm{eV}$. Although it is very close to other calculated data $0.66 \mathrm{eV}[5]$ and $0.7 \mathrm{eV}[3]$, still far smaller than the experimental band gap due to the DFT-GGA scheme tends to under estimate slightly the bonding group-II oxide polymorphs $[3,5]$.

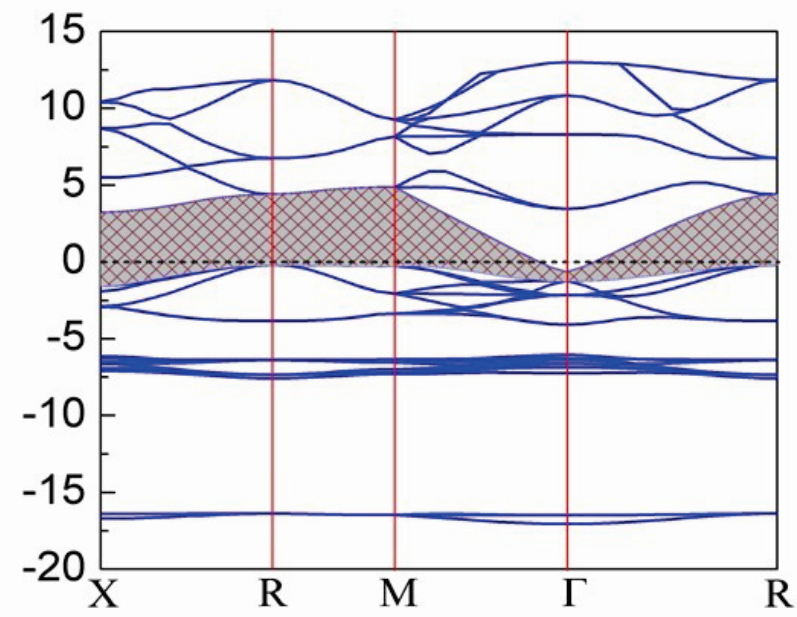

Figure 2. (Color online) Energy band structure of rocksalt CdO calculated within the DFT-GGA framework. The shaded region indicates the fundamental gap. The valence band maximum is chosen as energy zero.

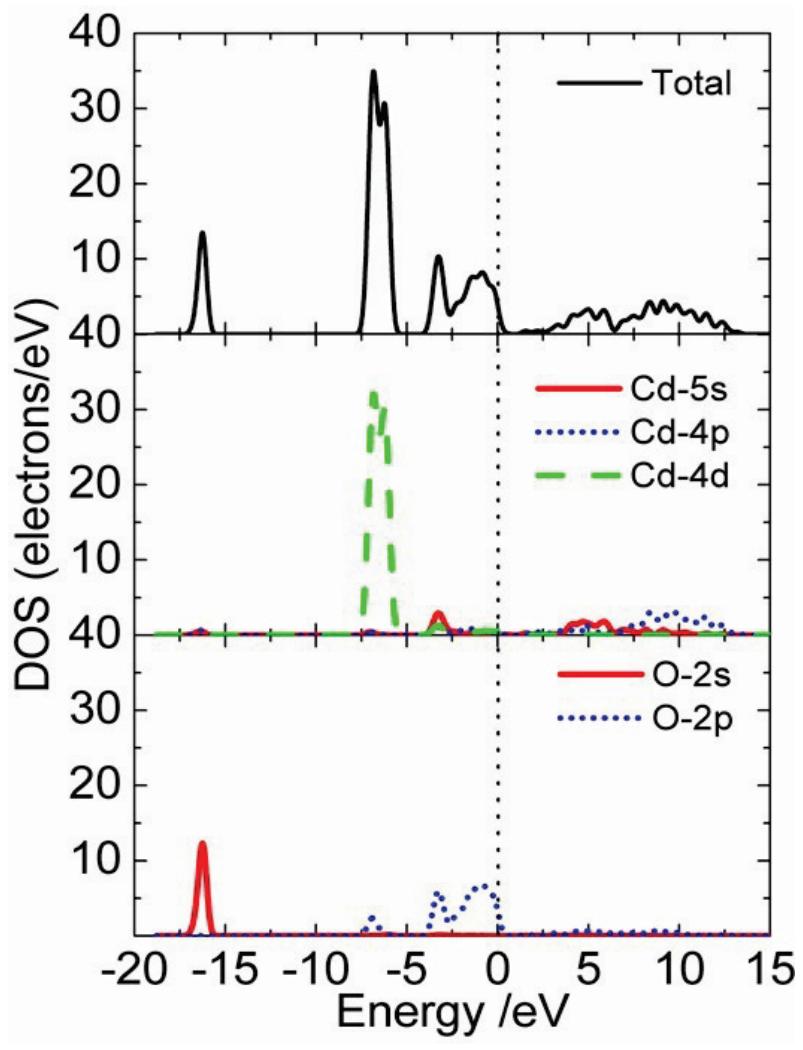

Figure 3. (Color online) Total and partial density of states of rocksalt CdO.

Nevertheless, GGA does correctly predict an indirect band gap for the rocksalt $\mathrm{CdO}$ with the maxima of the valence bands occur at the $\mathrm{R}$ point and the maxima lie 
above the conduction band minimum at the $\Gamma$ point, which results in negative indirect band gap of about $-0.51 \mathrm{eV}$ from $\mathrm{R}_{\mathrm{VB}}$ to $\Gamma_{\mathrm{CB}}$, indicates rocksalt $\mathrm{CdO}$ is a halfmetal material. In addition, rocksalt $\mathrm{CdO}$ may serve as a transparent conducting material because the bottom of conduction band in the pattern diffuse with a bandwidth $5.52 \mathrm{eV}$ fluctuates greatly than that of $1.38 \mathrm{eV}$ at the top of valence band.

By analyzing the partial density of states (PDOS) (see Figure 3), it was found that the energy bands between -17 and $-15.5 \mathrm{eV}$ mainly consist of $\mathrm{O} 2 \mathrm{~s}$ states. The energy bands at about $-7 \mathrm{eV}$ consist of $\mathrm{Cd} 4 \mathrm{~d}$ states shows a sharp peak due to its strong localization character. And it is found that the $\mathrm{O} 2 \mathrm{p}$ states have some admixture with the $\mathrm{Cd} s p$ states. Thus, $\mathrm{CdO}$ appears to have some covalent features. There are two peaks at about $-3.26 \mathrm{eV}$ and $-0.86 \mathrm{eV}$ in the upper valence bands. The lower one is mainly due to $\mathrm{O} 2 \mathrm{p}$ states hybridized with $\mathrm{Cd} 5 \mathrm{~s}$ and $4 \mathrm{~d}$ states. The upper peak is non-bonding $\mathrm{O}-2 \mathrm{p} \pi$. The conduction bands are mostly composed of Cd $5 \mathrm{~s}$ and $4 \mathrm{p}$ and show a weak hybridization with $\mathrm{O} 2 \mathrm{~s}$ and $2 \mathrm{p}$. Taken together, the energy state density curve near the Fermi level mainly come from the $\mathrm{Cd} 4 \mathrm{p}$ and $\mathrm{O} 2 \mathrm{p}$, which determine the type of charge carrier and the properties of electric conduction for rocksalt $\mathrm{CdO}$.

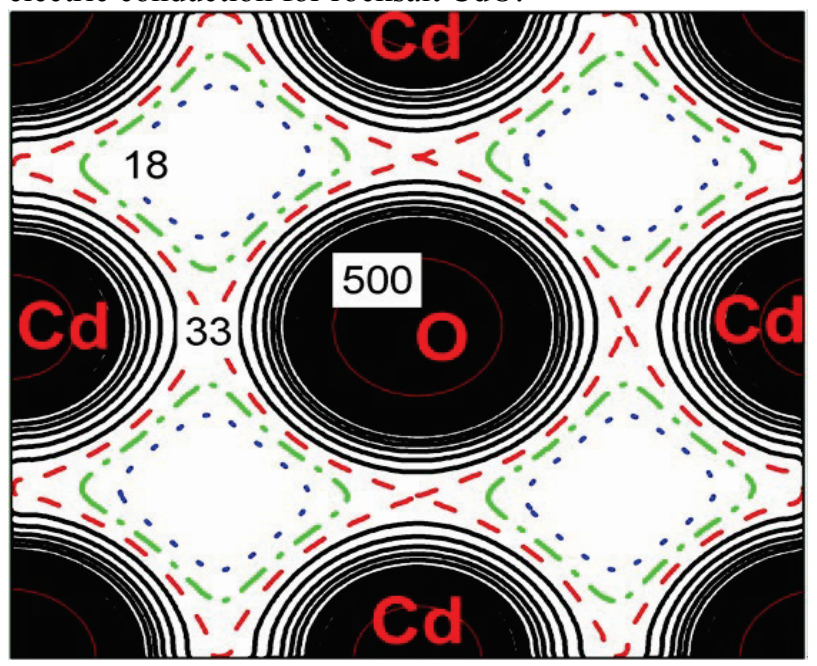

Figure 4. (Color online) Charge densities in the (002) plane of rocksalt $\mathrm{CdO}$.

In order to understand, goes a step further, the micromechanism of both atoms and interatomic, the charge densities of (002) plane was displayed in Figure 4 to serve as a complementary tool for achieving a proper understanding of chemical bonding. We also performed the Mulliken charge population for rocksalt $\mathrm{CdO}$ to understand bonding behavior. The Mulliken population results are given in Table 2 . The charge transfer from $\mathrm{Cd}$ to $\mathrm{O}$ is about $0.8 e$. It is relative smaller than the charge population both in $\mathrm{Cd}$ and $\mathrm{O}$ atoms. Therefore, we con- cluded that the bonding behavior of rocksalt $\mathrm{CdO}$ is a combination of weaker covalent and stronger ionic natures. And it is mainly contributed by both the $\mathrm{O} 2 \mathrm{p}$ and the $\mathrm{Cd} 4 \mathrm{~d}$ orbits when rocksalt $\mathrm{CdO}$ formed.

Table 2. The Mulliken charge population of rocksalt $\mathrm{CdO}$.

\begin{tabular}{lllllll}
\hline \multirow{2}{*}{ Species } & \multicolumn{6}{c}{ Charge population } \\
\cline { 2 - 7 } & $\mathrm{s}$ & $\mathrm{p}$ & $\mathrm{d}$ & $\mathrm{f}$ & Total & Charge $(e)$ \\
\hline $\mathrm{Cd}$ & 0.52 & 0.69 & 9.99 & - & 11.2 & +0.8 \\
$\mathrm{O}$ & 1.90 & 4.90 & - & - & 6.8 & -0.8 \\
\hline
\end{tabular}

\subsection{Optical properties}

The linear response of the system to an external electromagnetic field with a small wave vector is measured through the complex dielectric function $\varepsilon(\omega)=$ $\varepsilon_{1}(\omega)+i \varepsilon_{2}(\omega)$, which is mainly connected with the electronic structures. The imaginary part $\varepsilon_{2}(\omega)$ of dielectric function is derived based on the definition of direct transition [16-18]:

$$
\begin{aligned}
& \varepsilon_{2}(\omega)=\frac{V e^{2}}{2 \hbar m^{2} \omega^{2}} \sum_{i j} \int\langle k i|\vec{p}| k j\rangle^{2} f(k i)(1-f(k j)) . \\
& \delta\left(E_{k i}-E_{k j}-\hbar \omega\right) d^{3} k
\end{aligned}
$$

where $\vec{p}$ is the dipole matrix, $\mid k i>$ and $\mid k j>$ are the conduction band and valence band wave functions corresponding to the $i$ th and $j$ th eigenvalue with crystal momentum $k . f(k i)$ and $E_{k i}$ are the Fermi distribution function and the energy of electron for the $i$ th state. The real part $\varepsilon_{1}(\omega)$ of the complex dielectric function can be obtained using the Kramers-Krönig relations [16-18]. All other optical constants, therefore, such as the refractive index, extinction coefficient, absorption spectrum, reflectivity and energy loss spectrum can be obtained using the $\varepsilon_{1}(\omega)$ and $\varepsilon_{2}(\omega)$. Those optical spectrums are more convenient associate with the microscopic model for the physic progress, demonstrate the luminescence mechanism of spectrum produced by electron transitions in different energy levels and then characterize the physics properties for materials much more.

As shown in Figure 5 , the calculated real part $\varepsilon_{1}(\omega)$ and imaginary part $\varepsilon_{2}(\omega)$ of complex dielectric function get on well with those reported in Ref. [5]. Especially the static dielectric function $\varepsilon_{1}(0)(7.19)$ tally with the value (7.20) very well, which suggests that the method in present work is practicable. As is stated above, however, the DFT-GGA scheme tends to underestimate slightly the bonding in the considered group-II oxide polymorphs $[3,5]$. Thus, it needs to revise by scissor operator [10], which can correct the band gap and has been successfully applied to investigate the band gap of $\mathrm{TiO}_{2}[19], \mathrm{GeO}_{2}$ [20], etc., to attain considerable accuracy in optical prop- 
erties. The modified results by scissor operator (set to $\left.1.75\left(\Delta=\mathrm{Eg}_{\text {exp }}-\mathrm{Eg}_{\mathrm{cal}}=2.3-0.55\right)\right)$ were plot with dotted lines in Figure 5 . The absorption edge is about $2.23 \mathrm{eV}$ correspondence with experimental band gap $2.3 \mathrm{eV}$ [21], i.e., it is necessary and reasonable to correct optical properties by scissor operator. In this way, we proposes a prediction value $\varepsilon_{1}(0)=4.75$. Unfortunately, to our knowledge, there is few experimental and theoretical data of $\varepsilon_{1}(0)$ for our comparison. The real parts $\varepsilon_{1}(\omega)$ increased rapidly with the increasing frequency where the photon energy is less than $2.82 \mathrm{eV}$. The calculated maximum of the $\varepsilon_{1}(\omega)$ is about 7.04. The imaginary part $\varepsilon_{2}(\omega)$ becomes steeper with an increasing photon energy, which could obtain a conclusion that the rocksalt $\mathrm{CdO}$ could be used as potential TCOS materials.

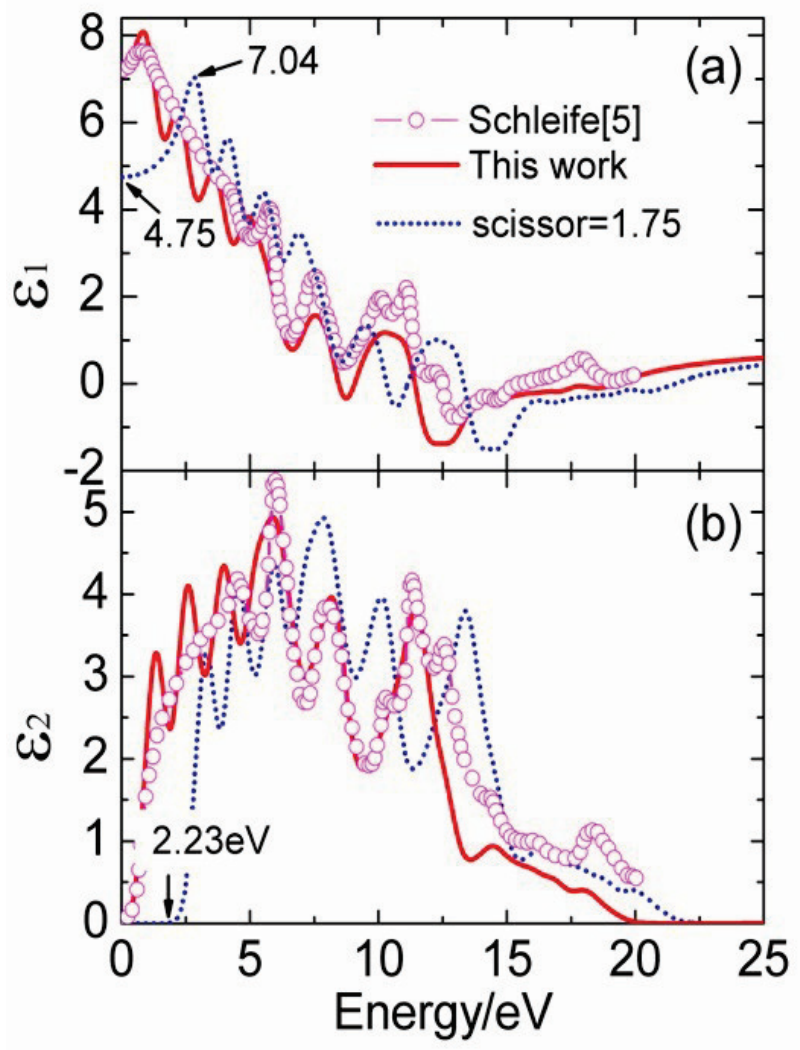

Figure 5. (Color online) Real part $\varepsilon_{1}(\omega)$ and imaginary part $\varepsilon_{2}(\omega)$ of the dielectric function $\varepsilon(\omega)$ of rocksalt $\mathrm{CdO}$.

\section{Conclusion}

In summary, we calculated the structural parameters, electronic structure, and optical properties for rocksalt CdO by means of the DFT within the GGA. Our structural parameters are in agreement with the previous experimental date. The electronic structures revealed that the top of the valence band and the bottom of the conduction band are decided by $\mathrm{O} 2 \mathrm{p}$ and $\mathrm{Cd} 4 \mathrm{p}$ states, respectively, and that $\mathrm{CdO}$ is a halfmetal material pre- sented a negative indirect band gap at $2.08 \mathrm{eV}$ in the $\mathrm{R}_{\mathrm{VB}}-\Gamma_{\mathrm{CB}}$ direction. Significantly, a more accurate prediction for $\varepsilon_{1}(0)$ of about 4.75 was obtained by using the scissor operator on the basic of our calculated band gap, suggesting that rocksalt $\mathrm{CdO}$ could be used as a potential TCOS materials. However, there is no experimental data available related to the rocksalt $\mathrm{CdO}$. Hence, careful experimental investigations are required in order to clarify the electronic band structure and optical properties of rocksalt $\mathrm{CdO}$ in detail.

\section{REFERENCES}

[1] J.S. Tse, D.D. Klug, S. Desgreniers, J.S. Smith, R. Dutrisac, EPL (Europhysics Letters) 86 (2009) 56001.

[2] P.D.C. King, T.D. Veal, A. Schleife, J. Zúñiga-Pérez, B. Martel, P.H. Jefferson, F. Fuchs, V. Muñoz-Sanjosé, F. Bechstedt, C. F. McConville, Phys. Rev. B 79 (2009) 205205.

[3] R.J. Guerrero-Moreno, N. Takeuchi, Phys. Rev. B 66 (2002) 205205.

[4] H. Liu, H. Mao, M. Somayazulu, Y. Ding, Y. Meng, D. Häusermann, Phys. Rev. B 70 (2004) 094114.

[5] A. Schleife, F. Fuchs, J. Furthmüller, F. Bechstedt, Phys. Rev. B 73 (2008) 245212.

[6] J.E. Jaffe, R. Pandey, A.B. Kunz, Phys. Rev. B 43 (1991) 14030.

[7] J. Zhang, Phys. Chem. Miner. 26 (1999) 644-648.

[8] M. Durandurdu, EPL (Europhysics Letters) 84 (2008) 66003.

[9] L.F.J. Piper, A. DeMasi, K.E. Smith, A. Schleife, F. Fuchs, F. Bechstedt, J. Zuniga-Perez, V. Munoz-Sanjosé, Phys. Rev. B 77 (2008) 125204.

[10] P. Dufek, P. Blaha, K. Schwarz, Phys. Rev. B 50 (1994) 7279 .

[11] M.D. Segall, P.J.D. Lindan, M.J. Probert, C.J. Pickard, P.J. Hasnip, S.J. Clark, M.C. Payne, J. Phys.: Condens. Matter 14 (2002) 2717.

[12] Y. Wang, J.P. Perdew, Phys. Rev. B 44 (1991) 13298.

[13] H.J. Monkhorst, J.D. Pack, Phys. Rev. B 13 (1976) 5188.

[14] Cimino, M. Marezio, J. Phys. Chem. Solids 17 (1960) 57-64.

[15] F. Birch, Phys. Rev. 71 ( 1947) 809.

[16] X.C. Shen, The Spectrum and Optical Property of Semiconductor, Science Press, Beijing, 1992.

[17] C.M.I. Okoye, J. Phys.: Condens. Matter 15 (2003) 5945.

[18] M.Q. Cai, Z. Yin, M.S. Zhang, Appl. Phys. Lett. 83 (2003) 2805.

[19] R. Asahi, Y. Taga, W. Mannstadt, A. J. Freeman, Phys. Rev. B 61 (2000) 7459.

[20] Q.J. Liu, Z.T. Liu, L.P. Feng, H. Tian, Solid State Sci. 12 (2010) 1748.

[21] F.P. Koffyberg, Phys. Rev. B 13 (1976) 447 